\title{
Alterations in Serum MicroRNA Profile During Hemodialysis - Potential Biological Implications
}

\author{
Dorota Trzybulska ${ }^{a}$ Dag Eckersten ${ }^{\mathrm{b}} \quad$ Aleksander Giwercman ${ }^{\mathrm{a}, \mathrm{c}}$ \\ Anders Christensson $^{b} \quad$ Christos Tsatsanis ${ }^{a, d}$ \\ aMolecular Reproductive Medicine, Department of Translational Medicine, Lund University, Malmö, \\ Sweden, bDepartment of Nephrology, Lund University, Skåne University Hospital, Malmö, Sweden, \\ 'Reproductive Medicine Centre, Skåne University Hospital, Malmö, Sweden, dDepartment of Clinical \\ Chemistry, School of Medicine, University of Crete, Heraklion, Greece
}

\section{Key Words}

MiRNA • Hemodialysis $•$ Comorbidities $•$ Cancer $・$ Fertility $・$ Inflammation

\begin{abstract}
Background/Aims: Hemodialysis implies significant alterations in the profile of serum components. microRNAs (miRNAs) are present in the human serum and are considered to target distant tissues where they can regulate gene expression, thus affecting homeostasis. Whether hemodialysis alters the profile of miRNAs in the serum is not known. Methods: miRNA profiling in serum samples collected before and after hemodialysis was performed using miRNA qPCR arrays. The results were subsequently validated in an independent group of 10 hemodialyzed men. miRWalk database was used to identify mRNAs targeted by the miRNAs the levels of which changed after hemodialysis. The list of mRNAs was analyzed using the DAVID and PANTHER classification systems to identify pathways controlled by these miRNAs. Results: miRNA profiling showed that the levels of the majority of circulating miRNAs were increased at least two-fold (115 out of 179 tested) while the levels of only five miRNAs were found at least two-fold lower after hemodialysis. Validation study confirmed the majority of the array results. Bioinformatics analysis of validated and significantly upregulated miRNAs revealed that gonadotropin-releasing hormone receptor, cell cycle and cell pluripotencyrelated pathways were targeted. Conclusion: Hemodialysis alters serum miRNA expression profile and this alteration may result in disruption of pathways contributing to subfertility and increased risk for cancer development being pathologies associated with hemodialysis.
\end{abstract}




\section{Introduction}

Chronic kidney disease (CKD) is a major problem worldwide affecting more than $10 \%$ of the general population [1]. CKD is divided into five stages with CKD 1 indicating preserved kidney function but with kidney damage (e.g. albuminuria) while CKD 5 indicates severe renal failure and is known as end stage renal disease (ESRD). The last phase of CKD-stage 5 means that the renal clearance of solutes is too low to survive without dialysis or a kidney transplant. Currently more than two million people worldwide require renal replacement therapy to sustain life [2].

Despite of removing uremic toxins, hemodialysis itself drastically activates leukocytes and complements. This induces, in an indirect way, diverse molecular changes which most frequently stimulate oxidative stress and inflammation being a signature of developing comorbidities [3-5]. Long-term hemodialysis has been associated with an array of pathologies including mainly cardiovascular diseases and risk of cancer [6-8]. In addition, male hemodialysis patients are frequently subfertile, partly attributed to disturbance of the hypothalamus-pituitary-gonadal (HPG) axis [5].

A class of molecules that regulate all biological processes underlying medical conditions are microRNAs (miRNAs). miRNAs are small non-coding RNAs (sncRNAs) which regulate target mRNAs, primarily by suppressing their expression. miRNAs target even up to $60 \%$ of mammalian messenger RNAs (mRNAs) regulating expression of genes post-transcriptionally, either by inhibiting protein translation or promoting mRNA degradation $[9,10]$. miRNAs as well as other sncRNAs are present in biofluids where they exhibit high stability and their expression pattern differs in pathological conditions. Therefore, they have emerged as a new class of biomarkers which can be used not only as diagnostic tools but also to monitor efficacy of treatments. miRNAs present in the extracellular space are associated with different transport forms increasing their stability and assisting in targeting distant organs and tissues. They are found encapsulated in microvesicles, trapped in apoptotic bodies, bound to high density lipoprotein (HDL) or to Argonaute family proteins [11]. Serum miRNAs not only passively carry information about intracellular processes occurring in the tissue of origin but they can be taken up by distant cells and tissues where they actively regulate gene expression $[11,12]$. As a result, change in the profile of serum miRNAs does not only reflect changes in a particular tissue but also mediates changes in the homeostasis of the organism.

It is unknown how exactly hemodialysis alters the profile of miRNAs in the serum. Three studies to date [13-15] present fragmented information on selected serum miRNAs in patients undergoing hemodialysis. Therefore, to address this gap, the aim of this study was to investigate if the process of hemodialysis alters the profile of miRNAs in serum in young men and identify a potential link with development of pathologies associated with hemodialysis.

\section{Materials and Methods}

\section{Study design and patients}

This study was approved by the Regional Ethics Committee at Lund University, Sweden (No. LU 541/2008), and all subjects provided written informed consent to participate in the study. The study adheres to the Declaration of Helsinki.

For miRNA profiling serum samples were collected from three men: 1) age 43, time on dialysis 74 months, unknown kidney disease, 2) age 44, time on dialysis 25 months due to polycystic kidney disease, and 3 ) age 43 , time on dialysis 28 months due to polycystic kidney disease. They were dialyzed on high flux HD filter Polyflux 21 (Gambro, Lund, Sweden). Treatment time was four hours three times a week. None of the patients were taking any immunosuppressive drugs. Two serum samples were obtained midweek, before and after the hemodialysis treatment. All the predialysis samples were drawn between 8-9 a.m.

For validation 10 men with ESRD on hemodialysis were consecutively enrolled in October 2017 


\section{Cellular Physiology Cell Physiol Biochem 2018;46:793-801 \begin{tabular}{l|l|l} 
and BOI: 10.1159/000488737 & $\begin{array}{l}\text { (c) 2018 The Author(s). Published by S. Karger AG, Basel } \\
\text { www.karger.com/cpb }\end{array}$
\end{tabular}}

Trzybulska et al.: Serum MicroRNA Changes During Hemodialysis

(two with diabetes type 1 and one with type 2, two with polycystic kidney disease, and the others with focal segmental glomerulosclerosis, hypertensive kidney disease, IgA nephropathy, rapidly progressive glomerulonephritis and interstitial nephritis). Their median age was 52.5 (IQR=10.3) years and median time on dialysis were 3.5 (4.3) years. The patients underwent HD treatment 3-5 times weekly for 12-15 hours per week. Samples analyzed in miRNA profiling were from patients hemodialyzed on high flux HD filter, Revaclear 400 Baxter (Baxter, Hechingen, Germany). Albumin and high-density lipoprotein (HDL) concentrations were measured before and after hemodialysis to account for serum concentration, particularly of miRNA carrying molecules. Mean HDL levels were $1.13 \pm 0.26$ vs. $1.23 \pm 0.49$ (medians $1.15(0.28)$ vs. $1.15(0.49)$ ) and mean albumin levels were $36.2 \pm 2.7$ vs. $37.6 \pm 4.2$ (medians 36.5 (4) vs. $37.0(4)$ ).

\section{RNA extraction}

Serum was centrifuged at $3000 \times g$ for 5 min to remove any insoluble components. Extracellular and circulating free RNA was isolated from identical serum volume $(200 \mu \mathrm{L})$ by using miRCURY'M RNA Isolation Kit-Biofluids (Exiqon, Vedbæk, Denmark) according to the manufacturer's instruction. Glycogen was added $(10 \mu \mathrm{g} / \mathrm{ml})$ to each sample to increase yield of RNA. For miRNA profiling a fixed amount of synthetic spike-ins (UniSp2, UniSp4 and UniSp5) was added at the beginning of RNA extraction according to the manufacturer's instructions, to monitor RNA loss during the procedure. For the validation analyses, cel-miR-39-3p was used as an external normalizer. RNA was extracted simultaneously from all samples.

\section{Profiling of miRNAs in serum}

Equal amount of RNA was reverse-transcribed into complementary DNA (cDNA) by using Universal cDNA Synthesis kit II (Exiqon). The mix of UniSp6 and cel-miR-39-3p RNA spike-ins was included in the reaction to control for CDNA synthesis inhibition. In order to identify whether hemodialysis alters the profile of serum miRNAs we analyzed the miRNA levels in individual serum samples from three patients using Serum/Plasma Focus microRNA PCR Panel (V4.M) (Exiqon) covering 179 miRNAs most frequently found in serum. Reactions were carried out using the ExiLENT SYBR Green (Exiqon) and run on a ViiA ${ }^{\mathrm{TM}} 7$ Real-time PCR System (Applied Biosystems, Foster City, USA). Cycling conditions recommended by the manufacturer for Applied Biosystems instruments were as follows: denaturation $95^{\circ} \mathrm{C} 10 \mathrm{~min}$, followed by 45 cycles at $95^{\circ} \mathrm{C}$ for $10 \mathrm{~s}$, and $60^{\circ} \mathrm{C}$ for $60 \mathrm{~s}$ with fluorescence data collection, and post qPCR melting curve analysis (ramp rate $1.6 \mathrm{C} / \mathrm{s}$ ). Raw data were processed with QuantStudio ${ }^{\mathrm{TM}}$ Real-time PCR Software (v.1.2). Baseline start and end cycles, and threshold were set manually according to guidelines predefined by Exiqon. All assays were checked for distinctness of melting curves.

\section{Validation}

In the validation step miScript II RT Kit (Qiagen, Hilden, Germany) with HiSpec Buffer was used. In order to validate results obtained after miRNA profiling, qPCR was carried out to detect most dysregulated miRNAs. Reactions were carried out in duplicates using the miScript SYBR Green PCR (Qiagen) and AriaMx Real-time PCR System (Agilent, Santa Clara, CA, USA).

\section{Data analysis}

Quality control was performed according to the manufacturer's instructions (Exiqon, Denmark) for: negative controls, outliers, spike-ins profiles and markers of hemolysis based on the results of the formula $\Delta \mathrm{Cq}$ (miR-23a-3p - miR-451a) where miR-451a is erythrocyte specific and miR-23a-3p is stably expressed and the result above seven indicates hemolysis [16]. Since there was possibility of some miRNAs to be eliminated from the serum during hemodialysis, for miRNAs in which one of the two samples (before or after hemodialysis) did not provide any PCR product (no Cq value) a Cq value of 40 was set, representing the lowest detectable amount. The differential expression of miRNAs before and after hemodialysis was calculated according to the following formula $2^{-(\triangle \mathrm{Cq})}$. External miRNAs were used to normalize results. Changes in expression are presented as medians.

For the validation analyses, Wilcoxon signed-rank test was performed to test differences between paired samples. Results are presented as median (interquartile range, IQR). $p$-value below 0.05 indicated statistical significance. The statistical analysis was done by using IBM SPSS Statistics software (v. 23.0, Chicago, IL). 
Bioinformatics analysis was performed in order to identify target pathways and functions of differentially expressed miRNAs. Validated mRNA targets of selected miRNAs were found using miRWalk v.2.0 (http:// zmf.umm.uni-heidelberg.de/apps/zmf/mirwalk2/miRpub.html) $[17,18]$. Since results of pathway analysis from different tools utilize different algorithms and nomenclature, we used two most recently updated and most frequently used classification systems: DAVID (The Database for Annotation, Visualization and Integrated Discovery, v.6.8) [19, 20] and PANTHER (Protein ANalysis THrough Evolutionary Relationships, v.11.1) [21] for discovery of related biological processes, molecular functions and cellular components and identification of signaling pathways potentially affected by proteins targeted by selected miRNAs. $p$-values were adjusted for multiple testing and the false discovery rate (FDR) was controlled by Benjamini-Hochberg procedure in Kyoto Encyclopedia of Genes and Genomes (KEGG) analysis.

\section{Results}

\section{Changes in miRNA profile after hemodialysis procedure}

The expression pattern of miRNAs in the serum of dialysis patients before and after hemodialysis was analyzed. The levels of most serum miRNAs were noticeably elevated after a hemodialysis session. Median expression of 115 miRNAs was elevated at least two-fold after hemodialysis including $71 \mathrm{miRNAs}$ elevated at least threefold, 24 miRNAs elevated at least five-fold and 13 miRNAs elevated at least 10-fold (Fig. 1). Median expression of five miRNAs was at least two-fold lower in comparison to samples before hemodialysis (Fig. 1). The individual miRNAs affected are shown in Table 1.

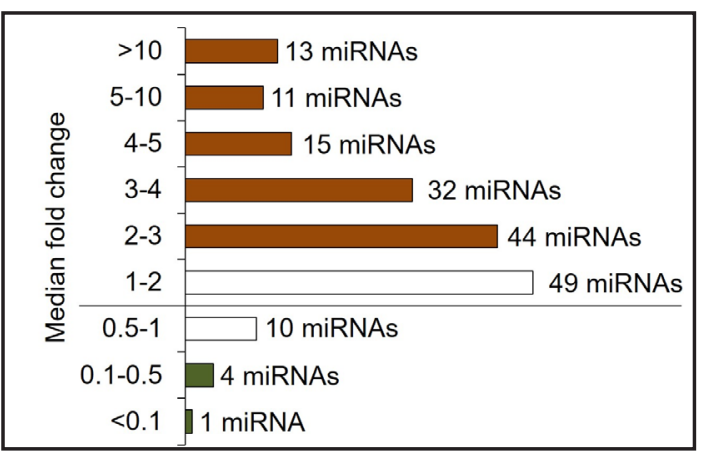

Fig. 1. A histogram presenting changes in the levels of serum miRNAs after a hemodialysis session in the exploratory study.

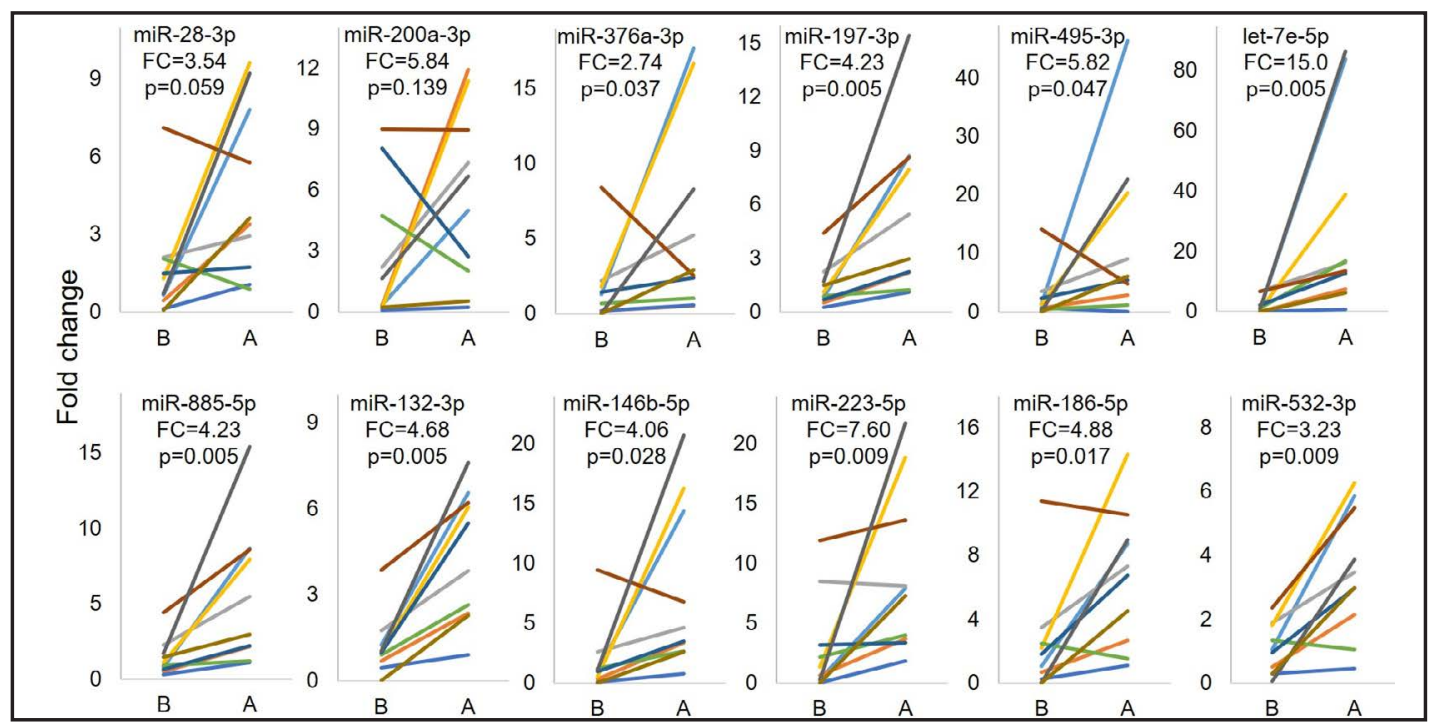

Fig. 2. Relative expression levels of statistically significantly changed miRNAs in sera collected before (B) and after $(A)$ hemodialysis session $(n=10)$. miRNAs with expression changes above 10 -fold were initially chosen among 179 miRNAs evaluated in the preliminary study. Levels of miRNAs are normalized to celmiR-39-3p. The difference before and after hemodialysis was tested by using the Wilcoxon signed-rank test. $\mathrm{p}<0.05$ indicates a statistically significant difference. FC, fold change. 


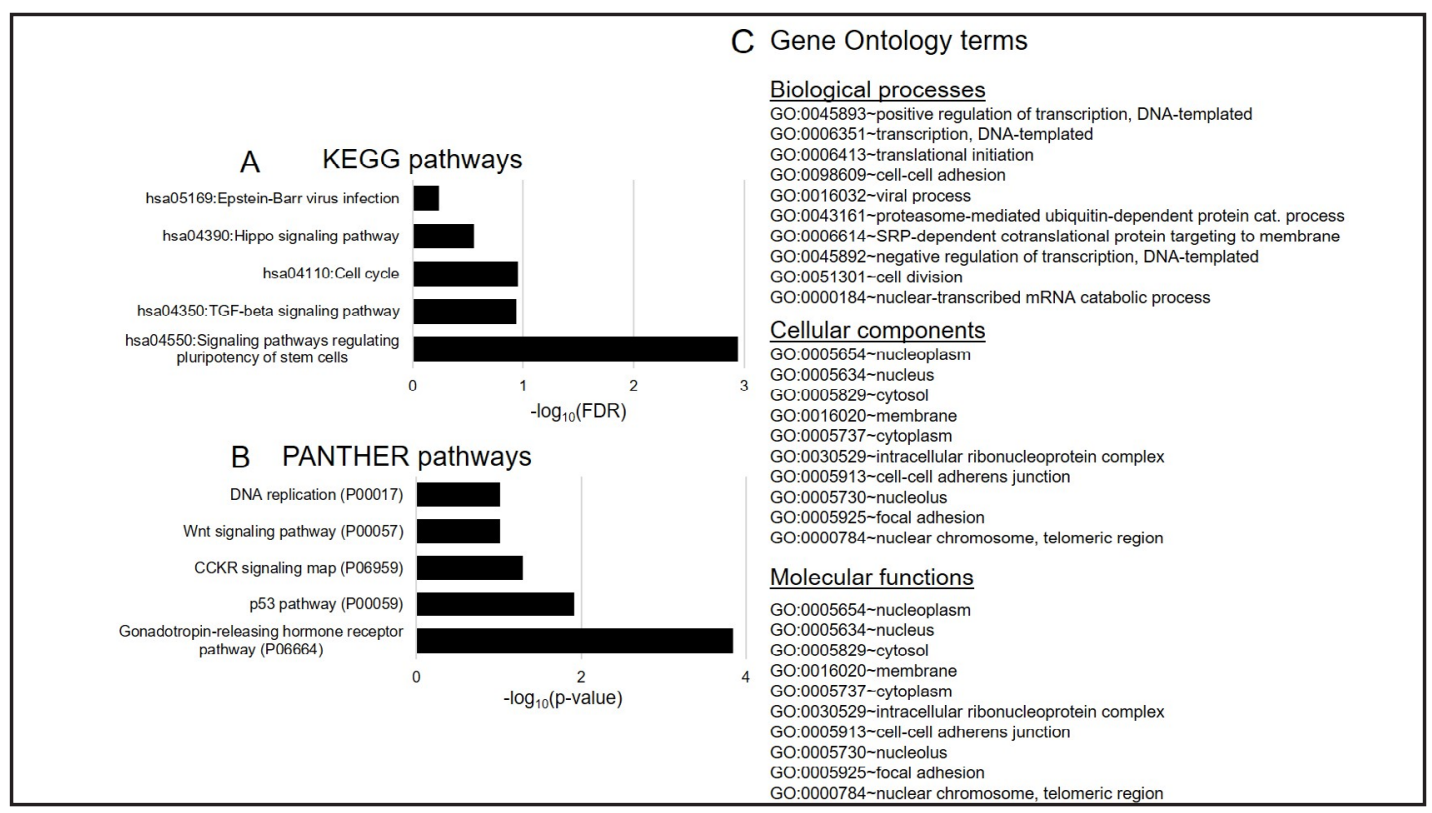

Fig. 3. Classification of genes potentially regulated by miRNAs at the end of hemodialysis procedure based on their function. (A) KEGG and (B) PANTHER pathways were analyzed using DAVID and PANTHER classification systems, respectively. Top 10 GO terms including biological processes, cellular components and molecular functions are listed in panel $\mathrm{C}$. The charts were built based on genes targeted by the miRNAs which displayed at least 10x higher expression after hemodialysis in the profiling study and were validated in an independent group showing statistically significant changes. The negative $\log _{10}$ of FDR is plotted on the $\mathrm{x}$-axis in KEGG analysis. The negative $\log _{10}$ of $\mathrm{p}$-value is plotted on the $\mathrm{x}$-axis in PANTHER analysis.

\section{Validation}

To confirm the trend observed in the profiling step, validation was performed for miRNAs which expression was at least 10-fold increased. The changes of miRNA levels in the validation analysis ranged between 2.7-15.0-fold, however not all miRNAs tested were found to be significantly affected by hemodialysis and fold changes were less pronounced than in the screening phase. Additionally, different basal miRNAs expression levels were observed at the inter-individual level. Detailed results are presented in Fig. 2.

Signaling pathway analysis for protein-coding genes controlled by miRNA upregulated during hemodialysis

Pathway analysis was performed based on genes targeted by miRNAs found elevated more than 10-fold (median value) after dialysis in the array analysis, and their increase was subsequently confirmed at the validation stage (Fig. 2). Analysis using miRWalk identified 2427 validated target genes of the selected miRNAs (hsa-miR-376a-3p, hsa-miR-197-3p, hsa-miR-495-3p, hsa-let-7e-5p, hsa-miR-885-5p, hsa-miR-132-3p, hsa-miR-146b-5p, hsamiR-223-5p, hsa-miR-186-5p, hsa-miR-532-3p). Bioinformatics analysis of these genes was performed using two classification systems, DAVID and PANTHER, to identify pathways potentially regulated by miRNAs targets. The results of Gene Ontology analysis of the target genes, including classes associated with biological processes, cellular components, and molecular functions are presented in Fig. 3.

\section{Discussion}

The main finding of this study was that after the hemodialysis session we observed changes in the pattern of miRNA expression profile in the serum. Changes in the levels of individual miRNAs varied from no change (considered as less than two-fold) to over 10- 
Table 1. Median and mean fold changes in the panel of serum miRNAs after single hemodialysis procedure

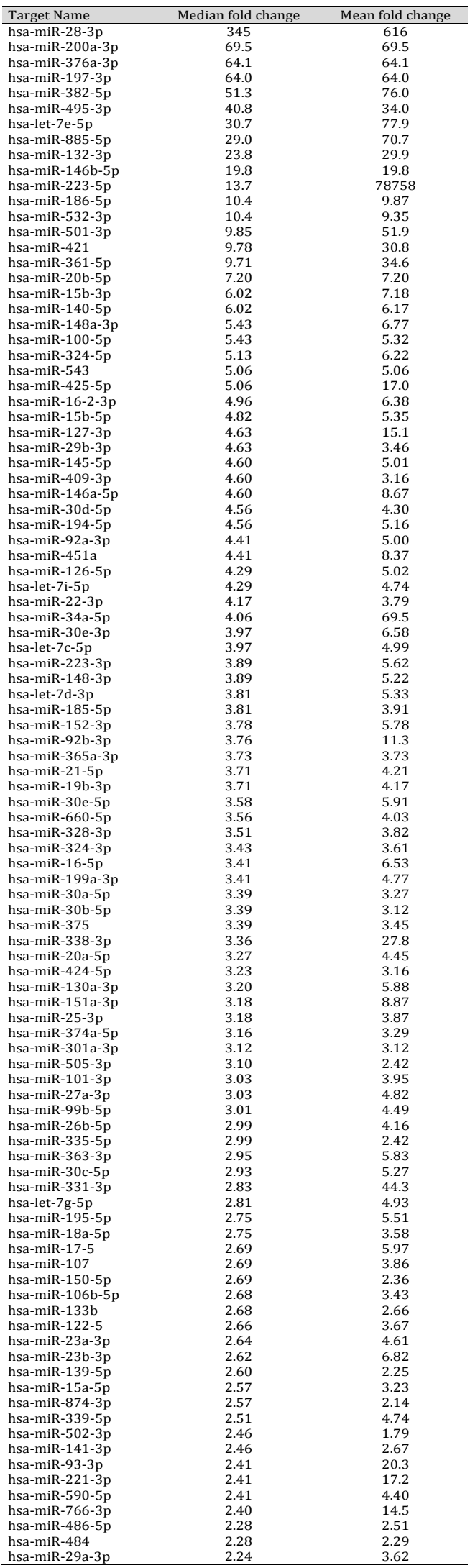

\begin{tabular}{|c|c|c|}
\hline Target Name & Median fold change & Mean fold change \\
\hline hsa-miR-33a-5p & 2.22 & 2.44 \\
\hline hsa-miR-191-5p & 2.20 & 3.49 \\
\hline hsa-miR-142-3p & 2.19 & 2.74 \\
\hline hsa-miR-378a-3p & 2.17 & 2.23 \\
\hline hsa-miR-423-3p & 2.16 & 5.77 \\
\hline hsa-miR-652-3p & 2.16 & 3.35 \\
\hline hsa-miR-24-3p & 2.13 & 4.15 \\
\hline hsa-miR-144-3p & 2.13 & 2.23 \\
\hline hsa-miR-376c-3p & 2.13 & 3.39 \\
\hline hsa-miR-140-3p & 2.10 & 4.93 \\
\hline hsa-miR-93-5p & 2.09 & 2.65 \\
\hline hsa-miR-28-5p & 2.09 & 32.3 \\
\hline hsa-miR-103a-3p & 2.04 & 2.98 \\
\hline hsa-miR-362-3p & 2.03 & 4.07 \\
\hline hsa-miR-454-3p & 2.01 & 3.66 \\
\hline hsa-miR-222-3p & 1.99 & 2.77 \\
\hline hsa-miR-199a-5p & 1.99 & 1.42 \\
\hline hsa-miR-193a-5p & 1.97 & 2.35 \\
\hline hsa-miR-130b-3p & 1.93 & 2.57 \\
\hline hsa-miR-126-3p & 1.92 & 2.73 \\
\hline hsa-let-7b-5p & 1.92 & 3.26 \\
\hline hsa-miR-208a-3p & 1.92 & 1.92 \\
\hline hsa-let-7d-5p & 1.91 & 1.91 \\
\hline hsa-miR-32-5p & 1.87 & 3.60 \\
\hline hsa-miR-339-3p & 1.84 & 5.23 \\
\hline hsa-miR-133a-3p & 1.83 & 1.89 \\
\hline hsa-let-7a-5p & 1.83 & 3.81 \\
\hline hsa-miR-106a-5p & 1.80 & 2.90 \\
\hline hsa-miR-192-5p & 1.80 & 1.64 \\
\hline hsa-miR-326 & 1.79 & 1.78 \\
\hline hsa-miR-99a-5p & 1.78 & 1.67 \\
\hline hsa-let-7b-3p & 1.77 & 8.04 \\
\hline hsa-miR-210-3p & 1.72 & 3.83 \\
\hline hsa-miR-136-5p & 1.71 & 2.13 \\
\hline hsa-miR-151a-5p & 1.71 & 3.73 \\
\hline hsa-miR-320a & 1.67 & 1.48 \\
\hline hsa-miR-143-3p & 1.64 & 1.94 \\
\hline hsa-miR-423-5p & 1.64 & 1.72 \\
\hline hsa-miR-27b-3p & 1.61 & 3.96 \\
\hline hsa-miR-320c & 1.60 & 1.76 \\
\hline hsa-miR-320b & 1.59 & 1.47 \\
\hline hsa-miR-26a-5p & 1.55 & 4.09 \\
\hline hsa-miR-154-5p & 1.52 & 15.2 \\
\hline hsa-miR-136-3p & 1.47 & 23.9 \\
\hline hsa-miR-144-5p & 1.46 & 3.22 \\
\hline hsa-miR-584-5p & 1.42 & 2.17 \\
\hline hsa-miR-125b-5p & 1.42 & 1.79 \\
\hline hsa-miR-629-5p & 1.39 & 1.39 \\
\hline hsa-miR-320d & 1.38 & 1.34 \\
\hline hsa-miR-342-3p & 1.36 & 1.57 \\
\hline hsa-miR-877-5p & 1.34 & 1.34 \\
\hline hsa-let-7-1-3p & 1.33 & 1.33 \\
\hline hsa-miR-128-3p & 1.30 & 1.30 \\
\hline hsa-miR-215-5p & 1.29 & 6.55 \\
\hline hsa-miR-18b-5p & 1.28 & 2.55 \\
\hline hsa-miR-29c-3p & 1.28 & 2.38 \\
\hline hsa-miR-205-5p & 1.26 & 15.5 \\
\hline hsa-let-7-5p & 1.19 & 1.26 \\
\hline hsa-miR-181a-5p & 1.14 & 1.39 \\
\hline hsa-miR-497-5p & 1.13 & 1.12 \\
\hline hsa-miR-155-5p & 1.05 & 1.86 \\
\hline hsa-miR-10b-5p & 1.04 & 2.06 \\
\hline hsa-miR-1 & 1.03 & 1.03 \\
\hline hsa-miR-142-5p & 1.03 & 1.03 \\
\hline hsa-miR-1260a & 0.97 & 1.48 \\
\hline hsa-let-7f-5p & 0.93 & 0.93 \\
\hline hsa-miR-574-3p & 0.91 & 11.9 \\
\hline hsa-miR-485-3p & 0.88 & 7.59 \\
\hline hsa-miR-125a-5p & 0.88 & 2.64 \\
\hline hsa-miR-532-5p & 0.73 & 0.75 \\
\hline hsa-miR-2110 & 0.71 & 0.85 \\
\hline hsa-miR-425-3p & 0.70 & 1.35 \\
\hline hsa-miR-22-5p & 0.62 & 0.62 \\
\hline hsa-miR-483-5p & 0.61 & 0.95 \\
\hline hsa-miR-374b-5p & 0.44 & 1.47 \\
\hline hsa-miR-19a-3p & 0.29 & 1.25 \\
\hline hsa-miR-200c-3p & 0.26 & 0.28 \\
\hline hsa-miR-106b-3p & 0.20 & 6.59 \\
\hline hsa-miR-335-3p & 0.03 & 0.46 \\
\hline
\end{tabular}


fold change. Bioinformatics analyses revealed that these miRNAs targeted the gonadotropinreleasing hormone receptor (GnRHR) pathway, and pathways related to tumorigenesis and inflammation/angiogenesis. To the best of our knowledge this is the first report investigating the effect of hemodialysis on serum miRNA profile and providing a potential pathogenetic link to dialysis-associated diseases. Indeed, subfertility and increased risk for cancer and inflammatory/cardiovascular diseases are among the observed pathologies in hemodialysis patients.

Earlier independent reports had shown upregulation of miR-499, miR-21-5p, miR210, miR-122-5p and miR-885 in the serum following hemodialysis [13-15]. Our analysis confirmed upregulation of miR-21-5p, miR-210-3p, miR-122-5p and miR-885-5p while miR499 was not included in the panel analyzed, further supporting upregulation of miRNAs in the serum of hemodialysis patients signalized previously by Rivoli et al. [15].

Several studies showed dramatic elevation of cell-free nucleic acids after hemodialysis [22-24]. It was suggested that it can be an effect of apoptosis being a consequence of the direct contact of cells with membranes [22]. In addition, contact of neutrophils and other immune cells with hemodialysis membranes may trigger secretion of inflammatory miRNAs. Another potential reason for elevated miRNA levels could be binding of miRNA to proteins of molecular weight higher than 30-40 kDa which are not cleared by hemodialysis [13], but since the increase of most miRNAs was higher than the effect of hemoconcentration of the plasma due to removal of water, this hypothesis is less likely.

Since expression of some miRNAs was initially found over 10-fold higher or for one of them lower after hemodialysis, and given the fact that serum miRNAs can affect gene expression in distant tissues, we hypothesized that these changes may alter gene expression in target tissues and contribute to hemodialysis-related pathologies and co-morbidities. To determine signaling pathways affected by the miRNAs exhibiting the most pronounced change after hemodialysis we performed an in silico analysis. The results highlighted that target mRNAs encode proteins involved in transcriptional regulation, and are primarily localized in the nucleus and cytoplasm. We then performed bioinformatics analysis focused on biological functions of these proteins. The results revealed that the miRNAs found increased after hemodialysis might affect transcripts for proteins involved in GnRHR pathway (thus potentially contributing to subfertility), proteins involved in cell proliferation and pluripotency (thus contributing to tumorigenesis), and proteins involved in angiogenesis and inflammation/metabolic pathways such as TGF $\beta$.

Disturbances in the HPG axis have been reported among male patients on hemodialysis and are thought to contribute to subfertility [5]. It is known that changes in GnRHR pathway may result in fertility impairment $[25,26]$. It has been reported that in male patients on hemodialysis testicular volume, being a parameter of spermatogenesis, is significantly smaller partly explaining poor semen quality. However, the mechanism involved remains unclear [5]. Another pathway identified as dysregulated by the miRNAs increased during hemodialysis was the cholecystokinin receptor (CCKR) signaling pathway. A recent study in mouse models and human cells revealed that CCK and CCKR regulate mature sperm capacitation and subsequent fertilization capacity [27], suggesting an additional mechanism through which changes in serum miRNAs can affect fertility in male hemodialysis patients.

Bioinformatics analysis revealed that cell pluripotency, cell cycle and components of Hippo and p53 signaling pathways were affected by the miRNAs the levels of which increased after hemodialysis. The Hippo and p53 pathways are important in retaining the balance between cell division and apoptosis $[28,29]$ which, when disrupted, results in tumorigenesis. Similarly, cell cycle regulatory proteins and proteins regulating cell pluripotency also contribute to tumorigenesis. Cancer development is another risk in ESRD patients. The risk of renal cancer development can be up to three-fold higher compared to patients from general population without ESRD [6-8,30]. Another cause of malignancy in patients undergoing hemodialysis may be metabolic changes or immune dysfunction induced by dialysis [7].

Transforming growth factor beta (TGF- $\beta$ ) pathway was also identified as potentially affected by miRNAs found elevated in the serum after hemodialysis. This pathway is involved 
in immune cell regulation and TGF- $\beta$ alone is known to contribute to immunosuppression and it also contributes to wound healing. Patients on hemodialysis exhibit increased inflammation [31] and inflammatory diseases are associated with hemodialysis including cardiovascular and metabolic disorders [32-34].

The present study has limitations. Diurnal variation for miRNAs levels should be considered. However, since samples were collected between morning-noon time and single session was not longer than four hours we can assume that our observation was an effect of hemodialysis and not a physiological change through the time, since diurnal variation occurs mainly between day and night and the change is not more than two-fold [35]. Another limitation arises from discrepancies in fold change between the screening and validation studies. In this study we aimed mainly at evaluation of hemodialysis effect on miRNAs expression, thus no exclusion criteria were used regarding the underlying diseases and their severity in both phases of the study, which might have influenced the results. Additionally, at the array analysis stage we opted to analyze individual samples as opposed to pooled samples to avoid interference from inter-individual differences, which were indeed observed at the validation stage. On the other hand, analyzing individual samples may be the reason for not being able to validate all array findings at the validation stage.

In conclusion, this study revealed that a large fraction of serum miRNAs changed upon hemodialysis altering the pattern of miRNAs present in the serum of these patients. Even though such change may be transient, the frequency of hemodialysis treatments and therefore of miRNA profile changes may have detrimental effects on the organism homeostasis. Bioinformatics analysis identified GnRHR-related and cancer-related pathways potentially affected by these changes, supporting that male subfertility and cancer, known to be increased in hemodialysis patients, may be causally linked to changes in serum miRNAs.

\section{Acknowledgements}

We would like to thank Gun-Britt Eriksson, Mona Hassan Al-Battat at the Wallenberg Laboratory and Bodil Jönsson at the Dialysis Department.

This article is part of the ReproUnion collaborative study, co-financed by the European Union, Interreg V ÖKS (NYPS 20200407), the Swedish Kidney Association, Stiftelsen för njursjuka, the Faculty of Medicine at Lund University, Research Funds of Region Skåne, and the Research Fund of Malmö University Hospital.

\section{Disclosure Statement}

The authors declare no conflict of interest exists.

\section{References}

1 Jha V, Garcia-Garcia G, Iseki K, Li Z, Naicker S, Plattner B, Saran R, Wang AY, Yang CW: Chronic kidney disease: global dimension and perspectives. Lancet 2013;382:260-272.

-2 Eggers PW: Has the incidence of end-stage renal disease in the USA and other countries stabilized? Curr Opin Nephrol Hypertens 2011;20:241-245.

3 Cheung AK, Sarnak MJ, Yan G, Dwyer JT, Heyka RJ, Rocco MV, Teehan BP, Levey AS: Atherosclerotic cardiovascular disease risks in chronic hemodialysis patients. Kidney Int 2000;58:353-362.

4 Oleskowska-Florek W, Polubinska A, Baum E, Matecka M, Pyda M, Pawlaczyk K, Breborowicz A: Hemodialysis-induced changes in the blood composition affect function of the endothelium. Hemodial Int 2014;18:650-656.

5 Shiraishi K, Shimabukuro T, Naito K: Effects of hemodialysis on testicular volume and oxidative stress in humans. J Urol 2008;180:644-650.

6 Liang JA, Sun LM, Yeh JJ, Sung FC, Chang SN, Kao CH: The association between malignancy and end-stage renal disease in Taiwan. Jpn J Clin Oncol 2011;41:752-757.

$\rightarrow 7$ Stengel B: Chronic kidney disease and cancer: a troubling connection. J Nephrol 2010;23:253-262. 


\section{Cellular Physiology Cell Physiol Biochem 2018;46:793-801 \begin{tabular}{l|l} 
and Biochemistry Publisher 10.1159/000488737 & $\begin{array}{l}\text { (c) } 2018 \text { The Author(s). Published by S. Karger AG, Basel } \\
\text { www.karger.com/cpb }\end{array}$
\end{tabular}}

Trzybulska et al.: Serum MicroRNA Changes During Hemodialysis

8 Lowrance WT, Ordonez J, Udaltsova N, Russo P, Go AS: CKD and the risk of incident cancer. J Am Soc Nephrol 2014;25:2327-2334.

-9 Bartel DP: MicroRNAs: genomics, biogenesis, mechanism, and function. Cell 2004;116:281-297.

10 Friedman JM, Jones PA: MicroRNAs: critical mediators of differentiation, development and disease. Swiss Med Wkly 2009;139:466-472.

11 Turchinovich A, Samatov TR, Tonevitsky AG, Burwinkel B: Circulating miRNAs: cell-cell communication function? Front Genet 2013;4:119.

12 Vickers KC, Palmisano BT, Shoucri BM, Shamburek RD, Remaley AT: MicroRNAs are transported in plasma and delivered to recipient cells by high-density lipoproteins. Nat Cell Biol 2011;13:423-433.

13 Martino F, Lorenzen J, Schmidt J, Schmidt M, Broll M, Gorzig Y, Kielstein JT, Thum T: Circulating microRNAs are not eliminated by hemodialysis. PLoS One 2012; 7:e38269.

14 Emilian C, Goretti E, Prospert F, Pouthier D, Duhoux P, Gilson G, Devaux Y, Wagner DR: MicroRNAs in patients on chronic hemodialysis (MINOS study). Clin J Am Soc Nephrol 2012;7:619-623.

15 Rivoli L, Vliegenthart AD, de Potter CM, van Bragt JJ, Tzoumas N, Gallacher P, Farrah TE, Dhaun N, Dear JW: The effect of renal dysfunction and haemodialysis on circulating liver specific miR-122. Br J Clin Pharmacol 2017;83:584-592.

16 Blondal T, Jensby Nielsen S, Baker A, Andreasen D, Mouritzen P, Wrang Teilum M, Dahlsveen IK: Assessing sample and miRNA profile quality in serum and plasma or other biofluids. Methods 2013;59:S1-6.

17 Dweep H, Sticht C, Pandey P, Gretz N: miRWalk--database: prediction of possible miRNA binding sites by "walking" the genes of three genomes. J Biomed Inform 2011;44:839-847.

18 Dweep H, Gretz N: miRWalk2.0: a comprehensive atlas of microRNA-target interactions. Nat Methods 2015;12:697.

19 Huang da W, Sherman BT, Lempicki RA: Systematic and integrative analysis of large gene lists using DAVID bioinformatics resources. Nat Protoc 2009;4:44-57.

20 Huang da W, Sherman BT, Lempicki RA: Bioinformatics enrichment tools: paths toward the comprehensive functional analysis of large gene lists. Nucleic Acids Res 2009;37:1-13.

21 Mi H, Huang X, Muruganujan A, Tang H, Mills C, Kang D, Thomas PD: PANTHER version 11: expanded annotation data from Gene Ontology and Reactome pathways, and data analysis tool enhancements. Nucleic Acids Res 2017;45:D183-D189.

-22 Korabecna M, Pazourkova E, Horinek A, Rocinova K, Tesar V: Cell-free nucleic acids as biomarkers in dialyzed patients. J Nephrol 2013;26:1001-1008.

23 Garcia Moreira V, de la Cera Martinez T, Gago Gonzalez E, Prieto Garcia B, Alvarez Menendez FV: Increase in and clearance of cell-free plasma DNA in hemodialysis quantified by real-time PCR. Clin Chem Lab Med 2006;44:1410-1415.

-24 Atamaniuk J, Ruzicka K, Stuhlmeier KM, Karimi A, Eigner M, Mueller MM: Cell-free plasma DNA: a marker for apoptosis during hemodialysis. Clin Chem 2006;52:523-526.

25 Millar RP, Lu ZL, Pawson AJ, Flanagan CA, Morgan K, Maudsley SR: Gonadotropin-releasing hormone receptors. Endocr Rev 2004;25:235-275.

26 Rispoli LA, Nett TM: Pituitary gonadotropin-releasing hormone (GnRH) receptor: structure, distribution and regulation of expression. Anim Reprod Sci 2005;88:57-74.

27 Zhou Y, Ru Y, Shi H, Wang Y, Wu B, Upur H, Zhang Y: Cholecystokinin receptors regulate sperm protein tyrosine phosphorylation via uptake of HCO3. Reproduction 2015;150:257-268.

28 Saucedo LJ, Edgar BA: Filling out the Hippo pathway. Nat Rev Mol Cell Biol 2007;8:613-621.

29 Surget S, Khoury MP, Bourdon JC: Uncovering the role of p53 splice variants in human malignancy: a clinical perspective. Onco Targets Ther 2013;7:57-68.

30 Christensson A, Savage C, Sjoberg DD, Cronin AM, O'Brien MF, Lowrance W, Nilsson PM, Vickers AJ, Russo P, Lilja $\mathrm{H}$ : Association of cancer with moderately impaired renal function at baseline in a large, representative, population-based cohort followed for up to 30 years. Int J Cancer 2013;133:1452-1458.

-31 Libetta C, Sepe V, Esposito P, Galli F, Dal Canton A: Oxidative stress and inflammation: Implications in uremia and hemodialysis. Clin Biochem 2011;44:1189-1198.

-32 Chirakarnjanakorn S, Navaneethan SD, Francis GS, Tang WH: Cardiovascular impact in patients undergoing maintenance hemodialysis: Clinical management considerations. Int J Cardiol 2017;232:12-23.

33 Merino A, Nogueras S, Buendia P, Ojeda R, Carracedo J, Ramirez-Chamond R, Martin-Malo A, Aljama P: Microinflammation and endothelial damage in hemodialysis. Contrib Nephrol 2008;161:83-88.

-34 Vaziri ND: Causes of dysregulation of lipid metabolism in chronic renal failure. Semin Dial 2009;22:644-651.

35 Heegaard NH, Carlsen AL, Lilje B, Ng KL, Ronne ME, Jorgensen HL, Sennels H, Fahrenkrug J: Diurnal Variations of Human Circulating Cell-Free Micro-RNA. PLoS One 2016;11:e0160577. 